Saavedra Fernández, Rocío. Sociedad civil y democracia: una aproximación al debate desde el capital social y la cultura política. GeoGraphos [En línea]. Alicante: Grupo Interdisciplinario de Estudios Críticos y de América Latina (GIECRYAL) de la Universidad de Alicante, 3 de julio de 2015, vol. 6, no 79, p. 151-179. [ISSN: 21731276] [DL: A 371-2013] [DOI: 10-14198GEOGRA2015.6.79].

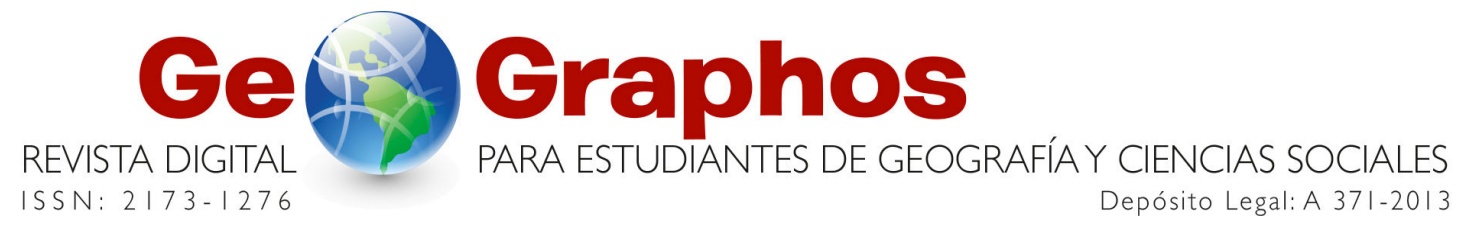

$<\underline{\text { http://web.ua.es/revista-geographos-giecryal }>}$

\title{
SOCIEDAD CIVIL Y DEMOCRACIA: UNA APROXIMACIÓN AL DEBATE DESDE EL CAPITAL SOCIAL Y LA CULTURA POLÍTICA
}

\author{
Rocío Saavedra Fernández \\ Doctoranda en Ciencias Políticas y de la Administración \\ Universidad de Santiago de Compostela (Santiago de Compostela, España) \\ Correo electrónico: saavedra.fdez@gmail.com
}

Recibido: 3 de febrero de 2015. Aceptado: 3 de julio de 2015

\section{RESUMEN}

La demostrada desafección política de la ciudadanía en un contexto sociopolítico en el que se cuestiona el papel del estado y se polarizan los pareceres acerca del protagonismo de la sociedad civil pone de relieve la necesidad de una reflexión acerca de los mecanismos que pueden garantizar el mantenimiento de los regímenes democráticos. Para ello proponemos un estudio de la relación existente entre la sociedad civil y la democracia, operacionalizando ambos conceptos a través del capital social y de la cultura política, respectivamente. Nos servimos así de un análisis de regresión lineal para identificar qué tipo de capital social se relaciona en mayor medida con la cultura política participante.

Palabras clave: Capital social, cultura política, democracia, España, sociedad civil. 


\title{
CIVIL SOCIETY AND DEMOCRACY: AN APPROACH TO DEBATE FROM CAPITAL AND POLITICAL CULTURE
}

\begin{abstract}
Political disaffection, when forging a context in which both the role of the state and the role of civil society are questioned, becomes a major issue. The risks it entails prompts a reflection on the mechanisms that work for the maintenance of democratic regimes. Finding ourselves in this context, we develop a study of the existing relationship between civil society and democracy, operationalizing each concept through social capital and political culture, respectively. Through a linear regression analysis, thus, we aim to identify which type of social capital is related to a greater extent to participant political culture.
\end{abstract}

Key words: Social Capital, Political Culture, Democracy, Spain, Civil Society.

\section{SOCIEDADE CIVIL E DEMOCRACIA: UMA ABORDAGEM PARA O DEBATE SOBRE O CAPITAL SOCIAL E CULTURA POLITICA}

\section{RESUMO}

O desafeto político, ao forjar um contexto no que tanto o papel do Estado e o papel da sociedade civil são questionados, torna-se uma questão de máxima relevância. Os riscos que implica dito desafeto exige uma reflexão sobre os mecanismos que trabalham pela manutenção dos regimes democráticos. Encontrando-nos neste contexto, pelo tanto, desenvolvemos um estudo sobre a relação existente entre a sociedade civil e a democracia, operacionalizando cada conceito através do capital social e da cultura política, respectivamente. Através de uma análise de regressão linear, assim, nosso objetivo é identificar qual é o tipo de capital social que está relacionado a um maior grau de cultura política participante.

Palavras chave: Capital social, cultura política, democracia, Espanha, sociedade civil.

\section{INTRODUCCIÓN}

A través de este trabajo buscamos indagar acerca de la relación existente entre dos conceptos clave de la Ciencia Política: el de sociedad civil y el de democracia. Además, y de manera transversal, queremos poner en valor el poder explicativo del concepto de capital social; concepto que, debido a la vaguedad con la que se ha definido hasta ahora, no parece haber alcanzado el puesto que merece en la disciplina.

La importancia de un estudio exhaustivo de la sociedad civil se entiende en el marco del mantenimiento de las democracias. Es cierto que la relación entre ambas nociones ha sido discutida de manera más o menos explícita desde tiempos de Tocqueville, Hegel o Gramsci. Retomar esta cuestión hoy en día, sin embargo, no es una decisión caprichosa, sino que responde al interés de encontrar una posible solución a la llamada crisis de la democracia, la cual se refleja en la manifiesta desvinculación política de la ciudadanía (Newton y Norris, 2000; Bouza Álvarez, 2013; Montero y Torcal, 2013). 
Para ello tomaremos como caso el de España, un país en el cual las encuestas muestran una severa desafección política en la ciudadanía. Y es que distintos barómetros de opinión evidencian un claro descenso de la confianza en las instituciones públicas, frente a un aumento considerable de la confianza en las fuerzas del orden (CIS, 2014: barómetro 3013). Este hecho suscita muchas dudas: ¿se está erosionando el sentir democrático? ¿Es esta desafección política el caldo de cultivo idóneo para una ruptura del régimen democrático?

Es ante estas preguntas, pues, cuando proponemos el estudio de la relación entre sociedad civil y democracia, como un medio para vislumbrar vías de prevención de una posible ruptura democrática. Así, al analizar el modo en que sociedad civil y democracia se relacionan, buscamos sentar las bases de políticas públicas orientadas a la promoción de la última.

Para ello comenzaremos con la elaboración de nuestra teoría, adentrándonos después en el modo de operacionalización de cada una de nuestras variables. A continuación, expondremos nuestro análisis estadístico, finalizando con la exposición de nuestras conclusiones.

\section{MARCO TEÓRICO}

La dicotomía público-privado está en auge en los días que corren. La crisis económica iniciada en 2007 ha abierto un debate acerca de la acción del Estado democrático (lo público) frente a la acción de la esfera económica (lo privado). La gravedad de la situación ha hecho que tal división se asevere en la mente de los ciudadanos, originándose así dos posturas encontradas: aquella que condena el reducido papel del estado y la excesiva presencia del sector privado y aquella que denuncia todo lo contrario, afirmando que el papel del estado es desmedido. Se exige de este modo, desde diferentes extremos, bien la limitación de la influencia de los mercados, bien la limitación del alcance del aparato estatal.

En lo que respecta a la democracia esta situación plantea una serie de dudas: ¿el crecimiento del sector privado se produce siempre en detrimento de lo público? ¿En qué modo afecta la presencia o ausencia de asociaciones privadas al estado por un lado y al régimen democrático por otro? ¿Merma la democracia con el aumento de las asociaciones que son ajenas a las instituciones o por el contrario se refuerza? Estas cuestiones, aunque se nos plantean originales a la luz de la actualidad, no son novedosas, sino que se han debatido a lo largo de la historia, generalmente a través del análisis de lo denominado como sociedad civil. Por ello, resulta imprescindible hacer un breve recorrido a través de los modos en los que se ha conceptualizado la sociedad civil al pasar de los años, como punto de partida para la construcción de nuestro propio concepto.

\section{Concepciones históricas de la sociedad civil y su relación con la democracia}

Algunos investigadores señalan que el primer teórico en referirse a la sociedad civil fue el liberal John Locke, a quien se le atribuye la ruptura con la idea tradicional de esfera pública unificada. Esto se debe a que el filósofo inglés afirmó que la sociedad aparece espontáneamente en el estado de naturaleza, teniendo una identidad propia que se contrapone y precede al gobierno, el cuál surge a posteriori como instrumento de perfeccionamiento (Peña, 2003). Otros, en cambio, cuestionan este argumento, asegurando que si bien Locke separa las nociones de sociedad civil y política de la de Estado o gobierno no es capaz de ilustrar con claridad cómo esa sociedad civil constituye un elemento no necesariamente 
político (Cohen y Arato, 2000).

Consideremos o no a Locke pionero en la teoría de la sociedad civil resulta relevante para nuestro estudio observar cómo la noción más general de sociedad civil estaba ya en sus inicios relacionada muy estrechamente con las de política y gobierno. De hecho, el que la sociedad civil se asumiese como elemento anterior al gobierno es una idea de la que nos haremos eco más adelante.

De manera similar destaca la aproximación hecha por Montesquieu, quien señala a la nobleza, el clero y las ciudades como poderes intermedios que limitan el poder de la monarquía (Peña, 2003). Esta identificación de las asociaciones como contrapeso al poder institucional está muy presente en las teorías contemporáneas de la democracia y la sociedad civil (Warren, 2001).

Hegel, por su parte, y ya considerado con firmeza por una serie de investigadores como el gran teórico de la sociedad civil (Cohen y Arato, 2000), añade una nueva cualidad a ésta, identificándola como ese espacio de intereses que trasciende tanto al núcleo familiar como a la unidad comunitaria o estatal.

En su obra Filosofia del Derecho [1821] Hegel conecta lo individual con lo colectivo, al hacer hincapié sobre la interdependencia entre las personas, quienes se hacen valer precisamente a través de sus relaciones. Afirma que los individuos, que tienen por fin particular su propio interés, no obtienen estabilidad sino a través de la universalidad (Hegel, 1968, p. 173-175). Además, su aproximación a la sociedad civil como sociedad burguesa también deja entrever elementos de vida asociativa y solidaridad, no reduciéndose en exclusiva a un asunto de mero egoísmo económico (Peña, 2003) e incorporando conceptos como el de Sittlichkeit, referido a las "normas de la vida pública de una sociedad (...) sostenidas por nuestra acción, y que no obstante ya estaban presentes" (Cohen y Arato, 2000, p. 122); concepto que ya apunta a la posterior aparición de teorías basadas en aspectos más abstractos como normas o valores culturales para explicar la acción colectiva o la misma instauración y mantenimiento de la democracia (Almond y Verba, 1970; Coleman, 1994).

Cabe destacar también el papel que Hegel le otorga al Estado, al cual considera el elemento clave que regulará, en base al interés general, los conflictos inherentes al sistema de necesidades e intercambio que caracteriza a la sociedad civil (Cohen y Arato, 2000). Este apunte nos acerca de un modo muy sutil a la idea de interdependencia, pues se asegura que la sociedad civil no es necesariamente capaz de discernir acerca del interés general, al igual que el estado no es capaz de promover un intercambio satisfactorio de necesidades a nivel individual.

Todos estos enfoques introducen algún elemento clave para la construcción de la idea de sociedad civil y su relación con el estado democrático. Sin embargo, si existe un acercamiento a la cuestión que es fundamental para el desarrollo de nuestro estudio ese es el de Alexis de Tocqueville.

Es Tocqueville [1835] quien, aun sin utilizar el término de sociedad civil, hace referencia explícita a la relación dada entre la existencia de asociaciones civiles y el estado democrático, entendiendo ambos como elementos diferenciados.

Es cierto, sin embargo, que lo que a priori puede parecer una defensa clara de lo favorable que es el asociacionismo para el estado democrático es en verdad un razonamiento mucho más complejo. Un estudio exhaustivo del contenido de La democracia en América, así como 
de su contexto histórico, nos lleva a entender que Tocqueville es bastante escéptico en lo que a la relación sociedad civil - democracia se refiere, no llegando a establecer una relación causal directa entre ambas (Whittington, 2001).

Y es que la proliferación de asociaciones en la América democrática le llama la atención precisamente porque sucede en un contexto de debilidad institucional. Tocqueville ve en la existencia de estos grupos una herramienta para el progreso que de ningún otro modo podría liderar el estado: “¿qué poder político podría llevar a cabo las innumerables pequeñas empresas que los ciudadanos americanos ejecutan a diario con ayuda de las asociaciones?" (Tocqueville, 1835, p. 142). Parece entonces que el autor entiende la presencia de estas asociaciones como un elemento que compensa la incapacidad del estado de proveer ciertos servicios. Sin embargo, Tocqueville nunca deja de referirse a la sociedad americana como democrática, lo que nos lleva a argumentar que para él la democracia es una condición social, y no tanto una forma de gobierno. En estos términos, pues, su análisis también sugiere la posibilidad de que la creación de asociaciones de este tipo suponga a la vez un impulso democrático y una amenaza a las instituciones políticas (Whittington, 2001).

Otros planteamientos que no debemos pasar por alto son los de Karl Marx y Antonio Gramsci. Consideramos adecuado referirnos a ellos ya que, aunque puedan parecer prescindibles, tratan un tema primordial para nuestra conceptualización: la sociedad civil como actividad económica.

El primero, Marx, entiende la sociedad civil única y exclusivamente como esfera de actividad económica contrapuesta a la esfera político-estatal, siendo el estado no un mediador de conflictos en pro del interés general, como apuntaba Hegel, sino un medio de perpetuación de la hegemonía de las clases dominantes, de la sociedad civil burguesa (Peña, 2003).

Por otra parte, y aunque partiendo de una concepción similar y una ideología hasta cierto punto equivalente a la de Marx, Gramsci dice reconocer la sociedad civil como "las nuevas formas de pluralidad y de asociación específicas de la sociedad civil moderna en las iglesias, sindicatos, instituciones culturales, clubes, asociaciones vecinales y especialmente partidos políticos" (Cohen y Arato, 2000).

Es importante llamar la atención sobre dos aspectos: el hecho de que Gramsci entiende la sociedad civil como separada tanto de la estructura económica como del aparato del estado y su propuesta de cómo las entidades que conforman la sociedad civil se consideran actores clave en la lucha por el poder, de manera que se les asigna una relevancia tal que se les relaciona con la existencia de movimientos sociales (Cohen y Arato, 2000; Peña, 2003).

Estos han sido, pues, algunos de los más destacados autores que han sentado las bases de la discusión acerca de la sociedad civil; discusión que a día de hoy continúa siendo encendida, tanto en lo que se refiere a su conceptualización como a su relación con otros fenómenos.

Esta diversidad de razonamientos pone de manifiesto cuáles son las grandes disputas teóricoconceptuales en torno a la idea de la sociedad civil: (I) la actividad económica como parte fundamental de la sociedad civil y (II) la sociedad civil como motor democrático. Serán, pues, estos dos ejes los que discutiremos a continuación, con el propósito de avanzar en la elaboración de nuestra teoría. 


\section{Actividad económica como parte fundamental de la sociedad civil}

La idea que subyace a la separación de las tres esferas, Estado, economía y sociedad civil, es la creencia en que sólo en esta última pueden existir relaciones libres entre individuos. Las conexiones enmarcadas en el Estado y la economía, por el contrario, estarían sujetas a una serie de coacciones y obligaciones, de ahí la necesidad de diferenciarlas tajantemente (Tenzer, 1992).

Creemos, sin embargo, que tal separación es teóricamente improductiva. En el marco de la investigación sobre los aspectos sociales que frenan o impulsan la democracia, no incluir a organizaciones económicas en la definición de sociedad civil reduciría los márgenes de investigación, excluyendo del análisis a grupos cuyas características los hacen tan potencialmente explicativos como a agrupaciones de índole religioso, social o deportivo. ¿O acaso no son las asociaciones de empresarios lugares donde se debaten libremente ideas y que sirven a una causa, donde se discurre en común y se puede transmitir actitudes democráticas? ¿Por qué razón habría que excluir a los círculos económicos, desde las grandes corporaciones hasta pequeñas cooperativas, claro ejemplo de participación y organización comunitaria?

Eliminar de nuestro estudio a una serie de asociaciones por el mero hecho de que entre sus objetivos se encuentre el del beneficio económico no es suficientemente convincente. Cabe la posibilidad de que lo que haga a un grupo o red ser fuente de actitudes democráticas no sea únicamente el fin de la asociación sino también el modo en el que se desarrollan las relaciones en él: verticalidad/horizontalidad, inclusión/exclusión, etc., rasgos que no son necesariamente ajenos a las asociaciones económicas (Puntam, 2002; Bowler, Donovan y Hanneman, 2003; Quintelier, 2008).

\section{Sociedad civil como motor de la democracia}

Las definiciones de democracia no se han hecho únicamente desde la perspectiva de las instituciones formales. El ciudadano, y especialmente sus actitudes, ha cobrado especial importancia en el desarrollo de teorías de la democracia, de manera que muchos politólogos han afirmado que el único garante de la democracia, tanto de su aparición como su mantenimiento, es la existencia de individuos democráticos en pensamiento (Farr, 2004).

De entre las muchas explicaciones sobre el papel fundamental del ciudadano en el desarrollo de la democracia destaca la presentada por Gabriel Almond y Sindey Verba, La Cultura Cívica (1970). Estos politólogos afirman que para la existencia de la democracia es necesario contar con la presencia de lo que denominan la cultura política participante, cuya tenencia supone la existencia de actitudes positivas hacia una serie de distintos elementos políticoadministrativos.

Tomando la cultura política participante como precondición para la existencia de la democracia, por tanto, situamos nuestro interés en la identificación de los fenómenos que promueven su aparición. Consideramos aquí la posibilidad de que uno de esos elementos sea la sociedad civil. Afirmaremos para nuestra teoría por tanto, y de manera general, que la existencia de una sociedad civil densa promueve y mantiene la democracia, por estar relacionada con la aparición de actitudes propias de la cultura política participante.

Esta teoría se sostiene desde el momento en que se entiende la sociedad civil como la esfera 
que materializa "la traslación inmediata [de las virtudes cívicas-democráticas] en la vida cotidiana de los individuos, en situaciones por tanto no directamente políticas" (Vázquez García, 2010, p. 98).

Es decir, la sociedad civil sería el punto intermedio entre el individualismo y la comunidad política. Y es que es en la asociación donde se demuestra el interés que cada individuo tiene por los asuntos comunes no necesariamente políticos, en el sentido en que descubre que él no es un ser aislado y que los otros pueden presuponer un límite u oportunidad para el alcance de sus objetivos; objetivos que al ser compartidos podrán ser alcanzados a través de la cooperación, tal y como apuntaba Hegel.

Consideramos así que una vez constituida esta unión las agrupaciones de la sociedad civil conseguirían, de manera transversal, influir en la cultura política del ciudadano, transformando su interés en la actividad común a pequeña escala en un compromiso político de ámbito más amplio. En esta línea se han señalado la capacidad de representación, los procesos de deliberación, la transmisión de habilidades sociales, la formación de opinión o la confianza como los rasgos característicos de la vida asociativa que tienen el potencial de socializar políticamente al individuo (Putnam, 2002; Warren, 2001).

Es así, pues, como se sostiene la idea de que la sociedad civil favorece la existencia de la democracia.

La otra premisa de nuestra teoría trata acerca de un aspecto sobre el que varios teóricos han querido llamar la atención: la posibilidad de que la capacidad de socializar políticamente no sea la misma en todas las asociaciones o redes (Van Deth et al, 1999).

Aquí sostendremos que ciertos sectores de la sociedad civil promueven actitudes democráticas en mayor medida que otros, en función de (I) el fin de la asociación y (II) el modo en que se dan las relaciones en dicha red.

Consideramos que el fin del grupo es clave desde el momento en que algunos grupos, por naturaleza, están orientados a los inputs del proceso político democrático. Nos referimos aquí a agrupaciones de tipo social con un fuerte énfasis en la acción cooperativa, así como a grupos de defensa de derechos de determinados colectivos. Este tipo de asociaciones centran su atención en objetivos externos, teniendo como intención la de crear o mantener ciertas condiciones sociales, y están en constante contacto con las instituciones públicas. Se trata de organizaciones que cuentan, sin duda, con un fuerte componente político, lo que las hace por definición agentes socializadores (Quintelier, 2008).

Además de la relación natural con las instituciones democráticas puede decirse también que el fin de un grupo fomenta más o menos la aparición de una cultura política participante según la amplitud de su objetivo. Esto es, y he aquí donde creemos reside la diferencia entre el sector económico y el sector social de la sociedad civil, las organizaciones cuyo fin reporta un beneficio para una amplia parte de la sociedad favorecen una socialización del tipo participativo en mayor medida que aquellos que buscan el beneficio de un colectivo muy concreto y cerrado. Esta idea se asemeja a la presentada por Putnam (2002) acerca de los grupos que tienden puentes (inclusivos) y los que vinculan (exclusivos).

Otro de los aspectos que serían clave en el desarrollo de valores democráticos en el núcleo de una red es el tipo de relaciones. Podría identificarse como rasgo clave, por ejemplo, la 
horizontalidad o la verticalidad (Putnam, 1993). Es de suponer, pues, que las redes inclusivas y horizontales se relacionarán en mayor medida con valores democráticos que las redes exclusivas y verticales, por funcionar estas acorde a un sistema de debate abierto donde la jerarquía se minimiza al máximo y las decisiones se toman desde la base. Asociaciones con tales características serían potenciales socializadores en actitudes democráticas.

Teniendo todo esto en cuenta, por tanto, esquematizamos nuestra teoría como muestra la siguiente figura:

\section{Figura 1. Teoría propuesta}

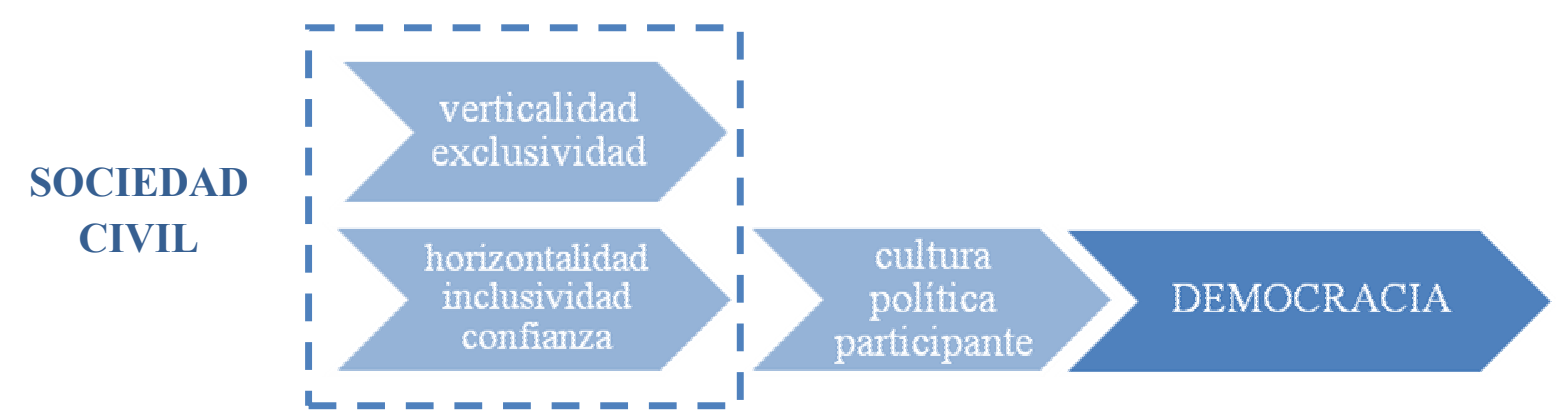

Fuente: Elaboración propia.

\section{ANÁLISIS: ACOTANDO EL CONTEXTO DE LA INVESTIGACIÓN}

Tal como hemos ido elaborando nuestra teoría cuenta con dos variables. Por una parte, la variable independiente sería la de la sociedad civil, la cual operacionalizaremos a través del capital social. Por otra, la democracia será operacionalizada a través de la cultura política participante. En este apartado ahondamos en ambos elementos.

\section{Capital social}

Una de las grandes dificultades que presenta la idea del capital social es la de la falta de consenso sobre su definición. Son muchas las formas en las que se ha definido, habiéndose entendido las veces como habilidad, recursos, relación, cultura o incluso como un conjunto todos esos elementos.

Algunos analistas han dado en organizar estas definiciones en tres bloques: el minimalista, donde se aglutinan las definiciones que enfatizan el carácter individual del fenómeno; el expansionista, referido a las definiciones que conectan el capital social con la acción colectiva, y el transicional, donde se encuentran aquellas definiciones lo suficientemente amplias como para conectar las dos tendencias anteriores, siendo, de hecho, las que promueven en cierto modo el salto de la perspectiva individual a la perspectiva de la acción colectiva (Ostrom y Ahn, 2003).

En este estudio nosotros entenderemos el capital social como la confianza y reciprocidad interiorizadas por un individuo. 
Renunciamos a entender el capital social como recursos objetivos y materiales, tal y como sugería Bourdieu (1986), por creer que la movilización de los recursos del otro por parte de un sujeto no es posible sin la existencia de una confianza y reciprocidad que enmarque la relación. Aun así, elegimos el término capital social no por una mera cuestión de resonancia, sino porque sí visualizamos la confianza como un elemento que puede invertirse para generar riqueza. Y es que tal como han recalcado autores como Coleman (1988) o Portes (1998), la confianza es un elemento clave para transformar determinados recursos, sean estos tangibles o intangibles, en otros que el individuo desea. Por ejemplo, es la confianza y la reciprocidad lo que permite un sencillo intercambio de bienes, el establecimiento de un préstamo entre amigos o incluso lo que facilita el acceso a determinados puestos de trabajo. Por este motivo elegimos hablar de capital social, pues estamos ante elementos susceptibles de ser invertidos, si bien reconocemos que, al igual que cualquier otro tipo de capital, puede no utilizarse en ese contexto de intercambio económico.

Por otra parte, mientras la gran mayoría de autores han optado por entender las redes como elementos que constituyen capital social (Jacobs, 1967; Coleman, 1988; Burt, 1992; Putnam, 1995; Paxton, 1999) nosotros rechazamos esta postura. Creemos que las redes no son capital social per se, sino manifestaciones del mismo, al igual que sugiere algún autor (Newton, 2001).

De todos modos, aceptamos la posibilidad de que grupos y asociaciones, en función de sus características propias, tales como cierre/apertura, verticalidad/horizontalidad, inclusión/exclusión.... refuercen y/o transformen el capital social. Afirmamos, pues, que el modo en que la interacción se produce en su seno puede moldear la confianza y normas de reciprocidad de sus miembros. No obstante, seguimos entendiendo que quien posee el capital social en última instancia es el individuo. Es cierto que "la confianza se da en el marco de la interacción, el cual está influenciado tanto por la personalidad como por el sistema social, no pudiendo ser asociada exclusivamente con ninguno" (Luhmann, 1979, p. 6). Sin embargo, es fundamental comprender que la confianza, si bien es moldeada tanto por aspectos individuales como institucionales, sólo existe en un sujeto para con el otro. Prueba de ello es la asimetría de confianza que se puede dar en una relación: mientras A confía plenamente en B, B puede no hacerlo en A. Así, al reconocer que la confianza depositada por cada individuo puede variar, estamos admitiendo que no es la estructura la poseedora de la misma, sino cada uno de los sujetos, que confiarán de un modo u otro en función de una serie de aspectos, entre los cuales sí se incluye el modo en que se configura dicha relación. Y es que es el individuo quien, a fin de cuentas, compone una comunidad, siendo él el portador o creador último de las normas que rigen sus interacciones con los demás (Brehm y Rahn, 1997).

Además, visualizar el capital social en el individuo nos permite huir de uno de los grandes problemas metodológicos encontrados tanto en los trabajos de Putnam (2002) como en las investigaciones que beben de su razonamiento (Paxton, 2002; Uslaner, 1999): la falacia ecológica. $\mathrm{Y}$ es que Putnam trabaja sobre atributos agregados a nivel estatal para concluir acerca de las correlaciones que se producen entre los mismos a nivel individual. Es decir, se dice que quienes viven en países con mayor capital social son más felices. Esto supone que los ciudadanos con más capital social son más felices. Sin embargo, esta conclusión nunca ha sido probada (Kadushin, 2012).

Por estos motivos optaremos por entender la confianza según la definición presentada por Diego Gambetta (1990, p. 217), quien la define en su modo más minimalista como el "nivel 
particular de la probabilidad subjetiva con la cual un agente evalúa si otro agente o grupo de agentes llevarán a cabo una determinada acción, antes de que él pueda comprobar tal acción (o independientemente de su capacidad de ser capaz de comprobarlo) y en un contexto en el cual afecta a su propia acción." La reciprocidad, estrechamente ligada a este concepto, la comprendemos como la posibilidad de que un actor responda, positiva o negativamente, a la acción que otro individuo ha llevado a cabo con respecto al primero. Se entremezcla aquí un juego entre confianza, expectativas y respuestas (Ostrom y Walker, 2005).

Creemos, al igual que otros autores de la disciplina, que operacionalizar la sociedad civil a través del capital social es una opción acertada (Paxton, 2002). Como hemos apuntado anteriormente, la asociación entre individuos requiere de la existencia de confianza y/o reciprocidad. Por ello, resulta lógico pensar que el estudio de la sociedad civil, de la asociación, ha de basarse en el análisis de los fenómenos que la sustentan, pues sólo así se podrá ahondar en su naturaleza. Creemos además que este planteamiento favorece la formulación de teorías complejas pero concretas, al hacer posible la conexión entre el nivel micro y macro, estrategia que varios autores han apuntado como fundamental a la hora de acercarse a este fenómeno (Lewis y Weigert, 1985; Misztal, 1996).

Distinguiremos, pues, cuatro tipos de capital social: estabilizador, cohesivo, tolerantemercantil y cooperativo. Esta clasificación se realiza siguiendo dos criterios: (I) la naturaleza de la motivación del individuo a confiar y (II) su predisposición a separarse y/o cohesionarse a otras personas o grupos.

\section{Naturaleza de la motivación}

Para identificar el tipo de motivación que subyace una relación de confianza y reciprocidad concreta nos basaremos en la propuesta de Bernard Williams (1990), quien distingue entre macro motivaciones y micro motivaciones. Las primeras se refieren a aquellas de carácter general, al hecho de que la confianza y reciprocidad guíen la mayoría de las acciones del individuo. Hablaremos de micro motivaciones cuando esas normas existan únicamente en el contexto de una acción puntual.

Distinguiremos, además, macro motivaciones egoístas, aquellas que no han de ser éticas o altruistas, sino que existen como resultado de la elección de confiar por temor a ser sancionado, y macro motivaciones no egoístas, las cuales sí responden a razones éticas o altruistas.

De manera similar hablaremos de micro motivaciones egoístas, cuando las bases que justifican la confianza sean las de un beneficio particular, y de micro motivaciones no egoístas, las cuales no esconden intereses más allá del altruismo.

A continuación (Fig. 2) se representa cómo proceder con la identificación de determinado tipo de confianza atendiendo a su motivación: 


\section{Figura 2. Procedimiento de clasificación de la dimensión de motivación}

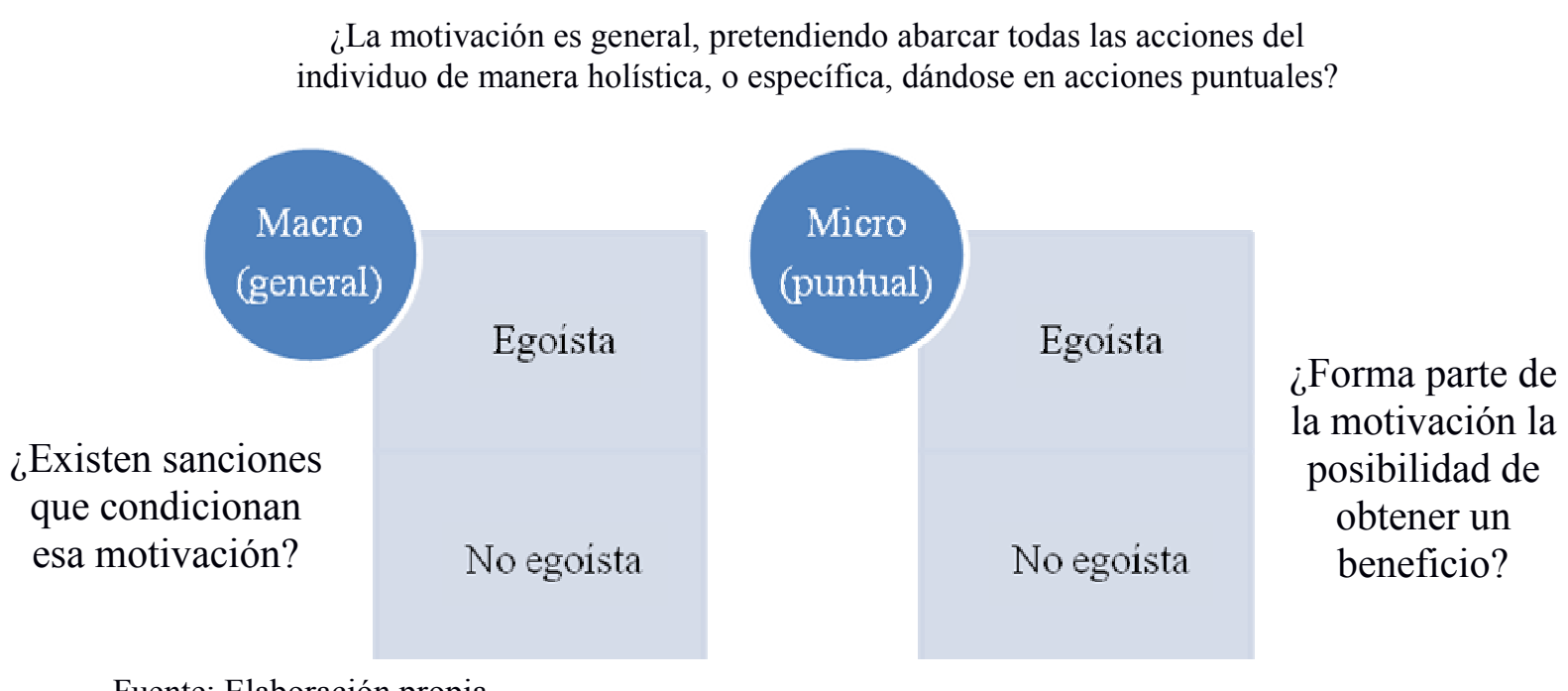

Fuente: Elaboración propia.

\section{Tendencia a la separabilidad y la cohesión}

Separabilidad y cohesión son aspectos a los que varios autores han hecho referencia, si bien desde aproximaciones variadas. Putnam (2002), por ejemplo, ha hecho hincapié en la necesidad de diferenciar entre aquellos grupos que tienden puentes (inclusivos) y aquellos que vinculan a sus miembros (excluyentes). Mark Granovetter (1973), por su parte, se ha referido a la confianza débil, la cual puede asociarse a la inclusividad o el tendido de puentes, frente a la confianza densa, relacionada con los grupos excluyentes.

Así, entenderemos que existen altos grados de separabilidad y cohesión cuando un actor profese confianza y normas de reciprocidad dentro de un grupo de actores bien definido y caracterizado por compartir atributos identitarios concretos entre sus miembros. Es el caso, por ejemplo, ya utilizado por Coleman (1988), del mercado de diamantes de Nueva York. Los individuos que forman parte de este grupo cuentan con normas de confianza y reciprocidad de tipo excluyente, puesto que estas existen sobre la base de que sus miembros son casi exclusivamente judíos.

Así, para identificar si la confianza de un individuo en determinado contexto es, pues, inclusiva o exclusiva, basta con preguntarnos lo siguiente:

Figura 3. Procedimiento para clasificación en la dimensión de separabilidad y cohesión

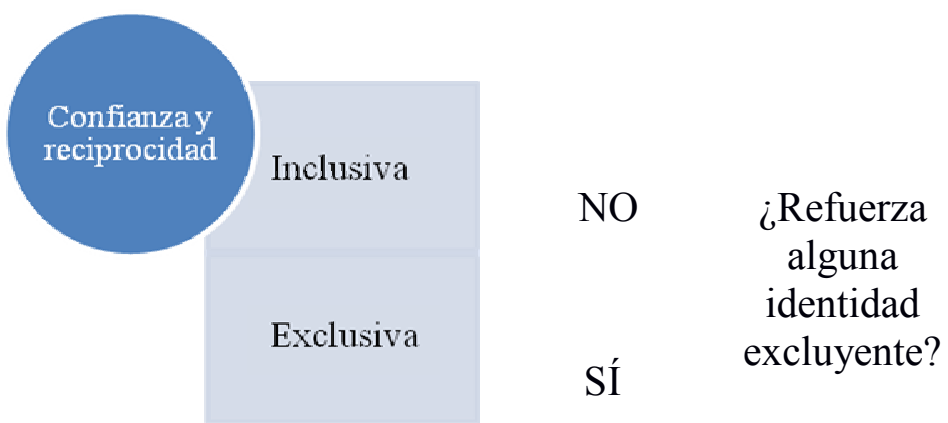

Fuente: Elaboración propia. 
Reconocemos los problemas que presenta esta clasificación. Por una parte, es posible encontrarse ante una combinación de ambos tipos de separabilidad y cohesión. Sucedería en el caso de las iglesias negras, en las cuales se excluye en función de origen y religión pero se incluye con respecto a otras variables como las de clase social (Putnam, 2002, p. 21).

Por otro lado, entendemos la limitación que supone que dicha clasificación se realice en numerosas ocasiones en base a generalizaciones, ya que la cantidad de información necesaria para realizar una clasificación exhaustiva de los tipos de confianza que maneja un individuo no suele ser de fácil acceso. No obstante, es necesario apuntar en torno a esta problemática la relevancia del estudio de redes desde los modelos matemáticos; área de estudio que se ha visto impulsada notablemente con la aparición de abundantes fuentes de datos provenientes de Internet (Kadushin, 2012; Rebola Pardo et al., 2013).

\section{Tipos de capital social}

Salvando los inconvenientes mencionados, y asumiendo los posibles límites que la puesta en práctica de esta clasificación pueda tener, distinguiremos entre los cuatro tipos de capital social mencionados según refleja el siguiente cuadro:

\section{Cuadro 1. Tipos de capital social en base a las dimensiones de motivación y separabilidad/cohesión ${ }^{1}$}

\begin{tabular}{|c|c|c|c|c|c|}
\hline & \multicolumn{4}{|c|}{ MOTIVACIÓN } \\
\hline & & \multicolumn{2}{|c|}{ MACRO } & \multicolumn{2}{|c|}{ MICRO } \\
\hline & & EGOÍSTA & $\begin{array}{c}\text { NO } \\
\text { EGOÍSTA }\end{array}$ & EGOÍSTA & NO EGOÍSTA \\
\hline \multirow{2}{*}{$\begin{array}{l}\text { SEPARABILIDAD } \\
\text { Y COHESIÓN }\end{array}$} & INCLUSIVO & $\begin{array}{l}\text { Tolerante - } \\
\text { mercantil } \\
\text { Networking }\end{array}$ & $\begin{array}{c}\text { Cooperativo } \\
\text { ONG }\end{array}$ & $\begin{array}{l}\text { Tolerante - } \\
\text { mercantil } \\
\text { Networking }\end{array}$ & $\begin{array}{c}\text { Cooperativo } \\
\text { Conocidos }\end{array}$ \\
\hline & EXCLUSIVO & $\begin{array}{l}\text { Estabilizador } \\
\text { Iglesia } \\
\text { católica } \\
\text { europea }\end{array}$ & $\begin{array}{l}\text { Cohesivo } \\
\text { Iglesia } \\
\text { EEUU }\end{array}$ & $\begin{array}{c}\text { Estabilizador } \\
\text { Sindicatos y } \\
\text { a. } \\
\text { profesionales }\end{array}$ & $\begin{array}{l}\text { Cohesivo } \\
\text { Amigos y } \\
\text { familia }\end{array}$ \\
\hline
\end{tabular}

Fuente: Elaboración propia.

La distinción entre estos cuatro tipos de capital social parte de la lectura de la obra Trust in Modern Societies: The search for the bases of social order (1996), escrita por Barbara A. Misztal y en la que se explora con exhaustividad el modo en el que la Sociología ha estudiado el fenómeno de la confianza a lo largo de los años.

\footnotetext{
${ }^{1}$ Hemos decidido incluir ejemplos en este cuadro por facilitar la comprensión de los tipos de capital social. No obstante, los mismos han de relativizarse. Es decir, no en todas las ocasiones, como se explicará más adelante, la participación en una ONG se entenderá como capital social cooperativo, ni la confianza depositada en la familia conformará capital social cohesivo.
} 
Además, la autora propone una tipología de la confianza atendiendo a la función que ésta tiene en la configuración del orden social (Snijders, 1997). Esta aportación, si bien a veces poco argumentada es sin duda el aspecto más destacado de su obra, e inspira en gran medida nuestra tipología del capital social.

De este modo, identificaremos como capital social estabilizador aquel que, tanto en el marco de las motivaciones macro como motivaciones micro, es egoísta y exclusivo. Este tipo de capital social se caracterizará, como apunta Mistzal, por sostener la predictibilidad, la confiabilidad y la legibilidad social. Esto es, las interacciones que se desarrollan como fruto de normas de confianza y reciprocidad estabilizadoras garantizan seguridad al individuo, puesto que simplifican los procesos sociales y generalizan las expectativas sobre el comportamiento del otro (Misztal, 1996, p. 98). Esto sucede a través, por ejemplo, de la puesta en marcha de rutinas, la existencia de un sistema de reputaciones que enmarca la reciprocidad o la memoria colectiva.

Es importante tener en cuenta que el razonamiento de Misztal cae en una contradicción, pues nos habla de esta confianza como calculada, razonada y consciente, mientras a la vez se refiere al concepto de Bourdieu (1988) de habitus para ilustrarla; concepto que se ha definido originalmente como el conjunto de disposiciones que orientan la acción de un individuo en función del espacio social que éste ocupa, siendo estos esquemas de actuación adquiridos inconscientemente.

Nosotros consideramos que lo adecuado es enmarcar el capital social estabilizador en el plano de la consciencia, del cálculo y la racionalidad. Así lo adecuado será desprendernos de la idea de habitus como preferencias involuntarias para visualizar la búsqueda de rutinas como una estrategia elegida para impulsar la predictibilidad del entorno.

Sería este tipo de confianza, pues, la que lleva al individuo a tomar parte en asociaciones profesionales o sindicatos en el nivel micro y a adscribirse, en el nivel macro, a instituciones religiosas como pudiera ser la Iglesia católica en el contexto europeo.

Por otra parte, la confianza que constituye lo que llamaremos capital social cohesivo no es ni estratégica ni racional. Se caracteriza, como indica el cuadro anterior, por ser exclusiva y no egoísta.

Impulsando relaciones de familiaridad, amistad y valores comunes, y operando a través de la internalización y el compromiso moral, esta confianza es fundamental a la hora de formar identidades, originando sentimientos de confianza así como obligaciones para con aquellos que forman parte de nuestra red de allegados cercanos (Misztal, 1996, p. 98). Entendemos, pues, que este tipo de confianza sí podría asociarse en mayor medida a un proceso inconsciente de identificación con un grupo, hecho que viene avalado por su carácter no egoísta.

Avanzando en la comprensión del capital social cooperativo, afirmamos que la confianza y normas de reciprocidad lo que componen son aquellas de carácter inclusivo y no egoísta.

Este tipo de confianza se convierte en un mecanismo de colaboración que promueve la cooperación independientemente de la existencia de sanciones o recompensas directas (Misztal, 1996, p. 99). 
Lo que caracteriza especialmente a este tipo de capital social es el hecho de la confianza que subyace en él es de carácter impersonal. Se trata de la confianza, pues, que impulsa la solidaridad, que no precisa de una identificación estricta con los gustos, valores o intereses del otro, sino de un sentimiento de identidad colectiva abstracto y relativamente amplio (Misztal, 1996, p. 100). Así, pues, se distingue del capital social cohesivo, que sí cuenta con un fuerte componente de identificación emocional en torno a una identidad concreta.

Encontramos esta confianza, por ejemplo, en la participación del individuo en actividades de índole asociativo como organizaciones no gubernamentales, o en la colaboración puntual desinteresada con personas que le son lejanas, por ejemplo, con lo que haríamos en llamar conocidos.

Por último, el capital social tolerante-mercantil responde a la existencia de confianza egoísta e inclusiva. Misztal incluye esta confianza en el marco de lo que hemos identificado como capital social cooperativo. No obstante, consideramos que existen diferencias lo suficientemente claras entre uno y otro como para distinguirlos. ${ }^{2}$

Mientras el capital social cooperativo tiene un carácter no egoísta que busca el beneficio no personal, sino, en todo caso, de una comunidad amplia e inclusiva, el capital social tolerantemercantil encuentra en la aceptación del otro, en la inclusividad, una fuente de beneficio.

La tolerancia se entiende aquí como "la aceptación reacia de una carga, aceptando aquello que uno no puede evitar, que no es lo suficientemente motivador" (Zeldin, 1994, p. 261, en Misztal, 1996). La multiplicidad de identidades se acepta y la confianza se construye en torno a esta realidad, entendiendo que ésta es necesaria para el provecho individual. Resulta sencillo comprender la naturaleza de esta confianza a través del ejemplo de lo que se ha dado en llamar networking.

Esta práctica, acuñada recientemente, se entiende como "una actitud ante la vida que refleja la forma en que nos relacionamos con los demás y que cuando es utilizada de forma inteligente, apropiada y profesional, constituye una poderosa y barata herramienta de marketing" (Calvo Muñoz y Rojas Llamas, 2009, p. 15-16). Es decir, esta práctica consiste en establecer relaciones de contacto, alimentando las normas de reciprocidad con otros actores, no porque compartamos determinados vínculos, sean afectivos sean identitarios, con ellos, sino porque tal relación puede reportarnos un beneficio. Así el networking o gestión de redes es una clara materialización de la confianza de tipo tolerante-mercantil.

Como se ha apuntado, el análisis del capital social se hará siempre en base al individuo, quien se entiende como contenedor de la confianza y las normas de reciprocidad. Sin embargo, cabe abordar la cuestión de si un individuo posee un solo tipo de capital social.

Creemos que un sujeto tendrá una serie de capitales sociales, pudiéndose dar múltiples combinaciones entre capital social cohesivo, cooperativo, tolerante-mercantil o estabilizador, micro y macro. Reconocemos también la posibilidad de que la distribución de los mismos no sea equilibrada, pudiendo pesar más en un individuo determinados tipos de confianza.

Esta situación, sin duda, presenta problemas de medición: ¿cómo determinamos con exactitud qué tipos de capital social posee cada individuo y cuáles pesan más en su persona?

\footnotetext{
${ }^{2}$ Misztal se refiere a esta confianza únicamente como tolerante. Nosotros, en cambio, preferimos añadir el término mercantil, puesto que éste denota con más exactitud la acción proactiva del individuo.
} 
El proceso ideal para determinarlo sería el de desarrollar una serie de entrevistas en profundidad en las que poder indagar acerca de los tipos de confianza que maneja un individuo, discerniendo con claridad las motivaciones subyacentes a cada uno. Para ello es fundamental que la perspectiva emic - la descripción términos de significado que el agente en cuestión hace de su propias prácticas - guíe la clasificación (Moreno Feliu, 2010).

He aquí donde reside la mayor limitación de nuestro trabajo. Realizar este tipo de entrevistas resulta inviable, dada la falta de recursos y la inadecuación de la información disponible en las bases de datos públicas.

No obstante, creemos que una aproximación básica al asunto, desde los datos de los que disponemos, sí arrojará luz sobre la cuestión que nos ocupa, señalando el camino sobre el cuál continuar en futuras investigaciones.

Para ello utilizaremos la base de datos que el Centro de Investigaciones Sociológicas proporciona para su estudio $\mathrm{n}^{\circ} 2.960$, de octubre de 2012, en la cual se recoge información acerca de la participación de cada encuestado en una serie de grupos, incluyendo partidos políticos, sindicatos o asociaciones de empresarios, colegios profesionales, parroquias o asociaciones religiosas, grupos deportivos, grupos culturales o de ocio, organizaciones de apoyo social o derechos humanos y asociaciones juveniles o estudiantes.

Así, pues, utilizaremos los datos proporcionados a través de esta pregunta para indagar acerca de la relación existente entre los tipos de capital social estabilizador y cooperativo y la cultura política. Es importante resaltar, sin embargo, que nosotros no haremos distinción entre la participación activa, la no activa o la antigüedad, sino que basaremos nuestro razonamiento únicamente en la pertenencia o no a determinados grupos.

De este modo asumiremos que los individuos encuestados poseen el capital social del tipo indicado en el siguiente cuadro en función de la organización a la que pertenecen:

\section{Cuadro 2. Clasificación del tipo de capital social que caracteriza al individuo que forma parte de las organizaciones mencionadas}

\begin{tabular}{|c|c|c|c|c|c|}
\hline & \multicolumn{4}{|c|}{ MOTIVACIÓN } \\
\hline & & \multicolumn{2}{|c|}{ MACRO } & \multicolumn{2}{|c|}{ MICRO } \\
\hline & & EGOÍSTA & NO EGOÍSTA & EGOÍSTA & NO EGOÍSTA \\
\hline \multirow{2}{*}{ 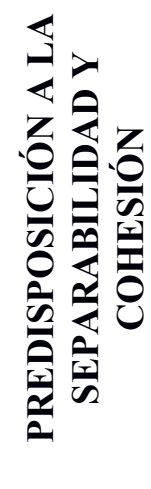 } & Inclusivo & $\begin{array}{l}\text { Tolerante - } \\
\text { mercantil }\end{array}$ & $\begin{array}{l}\text { Cooperativo } \\
\text { Org. de apoyo } \\
\text { social o derechos } \\
\text { humanos }\end{array}$ & $\begin{array}{l}\text { Tolerante - } \\
\text { mercantil }\end{array}$ & $\begin{array}{c}\text { Cooperativo } \\
\text { Grupo } \\
\text { deportivo }\end{array}$ \\
\hline & Exclusivo & $\begin{array}{l}\text { Estabilizador } \\
\text { Parroquia o } \\
\text { asociación } \\
\text { religiosa }\end{array}$ & Cohesivo & $\begin{array}{l}\text { Estabilizador } \\
\text { sindicato, } \\
\text { asociación } \\
\text { profesional }\end{array}$ & Cohesivo \\
\hline
\end{tabular}

Fuente: Elaboración propia. 


\section{Cultura política}

A la hora de conceptualizar la cultura política nos servimos de la propuesta hecha por Gabriel Almond y Sidney Verba en su obra La Cultura Cívica: estudio sobre la participación política democrática en cinco naciones (1970). Entenderemos, así, que la cultura política puede adoptar tres formas ideales: la parroquial, de súbdito y participante. Tal como sugieren, y desde una perspectiva micro, distinguiremos las tres en función de las disposiciones cognitivas, afectivas y evaluativas de cada individuo con respecto a cuatro elementos fundamentales: el sistema político, los procesos u objetos políticos que conforman los inputs (papel de las élites políticas, procesos de interacción y negociación entre dirigentes, programas o planes de gobierno), los objetos administrativos o logros entendidos como outputs (políticas públicas, imposición política, decisiones) y el rol que asume cada uno en este contexto político (1970, p. 30-32)

Para elaborar el índice de cultura política del que se servirían Almond y Verba, los politólogos realizaron una serie de encuestas y entrevistas en profundidad en las que se enfrentaba a cada individuo a una serie de cuestiones que esclarecerían el tipo de actitudes políticas del mismo. Aun si lo ideal a la hora de elaborar nuestro índice sería desarrollar nuestras propias encuestas, la falta de recursos nos obliga a recurrir a ficheros de datos y cerrados que, de algún modo, se aproximan a nuestros intereses. Así, tras haber observado los cuestionarios y tipos de datos que proporcionan estudios como la Encuesta Mundial de Valores (World Values Survey), la Encuesta Europea de Valores (European Values Study) o los barómetros del Centro de Investigación Sociológicas (CIS), concluimos que es esta última institución la que mejor responde a nuestras necesidades.

Tomaremos, pues, el fichero de datos perteneciente al Barómetro 2.960, de octubre de 2012. Los motivos fundamentales de esta elección son dos: por una parte el fichero contiene información no sólo de intención de voto sino también actitudes sobre el sistema político en general y, por otra, pregunta al encuestado acerca de su participación en una serie de grupos (asociaciones, sindicatos, grupos deportivos, partidos políticos...), lo que nos permite conectar el fenómeno de capital social con el de cultura política en el nivel que queremos: el individual.

Así, basándonos en dicho barómetro, elaboraremos nuestro índice de cultura política micro, tal como indica el siguiente cuadro:

\section{Cuadro 3. Elaboración de un índice de Cultura Político Micro desde el barómetro $n^{\circ} \mathbf{2 . 9 6 0}$ del CIS}

\begin{tabular}{|ccc|} 
& $\begin{array}{c}\text { Orientaciones afectivas, evaluativas y } \\
\text { cognitivas hacia... }\end{array}$ & Preguntas \\
& Sistema político en general & P1203, P1205 \\
CULTURA & Inputs & P13, P1701-P1714 \\
POLÍTICA & Outputs & P904, P1202 \\
MICRO & Rol de uno mismo & P1001, P1201, P1204, P1206, \\
& & P1501-P1504 \\
\hline
\end{tabular}

Fuente: Elaboración propia. 
Cada una de las variables que hemos tomado para la configuración de nuestro índice se ha reconfigurado, de manera que se ha asignado a cada respuesta un valor en una escala del 0 al 10 , donde 0 es la actitud correspondiente al tipo de cultura política parroquial, 5 es el tipo ideal de súbdito y 10 el tipo ideal de participante. De este modo, se agrega a través de la media aritmética los valores asignados a cada respuesta en una nueva variable, llamada Cultura política micro. De ahí obtendremos un valor en esa escala $0-10$, que indicará si las actitudes políticas de cada caso se aproximan más al tipo parroquial (0), súbdito (5) o participante (10).

Tras dicha recodificación cada caso contará con un valor de cultura política micro, de manera que la imagen general sería la que sigue:

\section{Cuadro 4. Distribución de frecuencias de la cultura política micro}

\begin{tabular}{|c|c|c|c|c|c|}
\hline & Válidos & Frecuencia & Porcentaje & $\begin{array}{l}\text { Porcentaje } \\
\text { válido }\end{array}$ & $\begin{array}{l}\text { Porcentaje } \\
\text { acumulado }\end{array}$ \\
\hline \multirow[t]{4}{*}{ Parroquial } & 0 & 1 & ,0 &, 0 & ,0 \\
\hline & 1 & 1 & , 0 & , 0 & , 1 \\
\hline & 2 & 174 & 7,0 & 7,0 & 7,1 \\
\hline & 3 & 425 & 17,1 & 17,1 & 24,2 \\
\hline \multirow[t]{4}{*}{ Súbdito } & 4 & 529 & 21,3 & 21,3 & 45,5 \\
\hline & 5 & 611 & 24,6 & 24,6 & 70,1 \\
\hline & 6 & 460 & 18,5 & 18,5 & 88,6 \\
\hline & 7 & 214 & 8,6 & 8,6 & 97,2 \\
\hline \multirow{4}{*}{ Participante } & 8 & 57 & 2,3 & 2,3 & 99,5 \\
\hline & 9 & 12 & ,5 &, 5 & 100,0 \\
\hline & 10 & 0 & , 0 & , 0 & \\
\hline & Total & 2484 & 100,0 & 100,0 & \\
\hline
\end{tabular}

Fuente: Elaboración propia.

Teniendo en cuenta de nuevo el significado de esta escala de cultura política, donde el valor 0 representa el tipo ideal de cultura política parroquial, el 5 la de súbdito y el 10 la de participante, podemos afirmar que la cultura política de los españoles gira en torno a la cultura política de súbdito. Hay, sin embargo, una tendencia hacia los valores inferiores, de manera que un $70 \%$ se incluye en el umbral $0-5$. Las tendencias participativas son menores, aunque no por ello inexistentes, y se reducen según se acercan al tipo ideal, concentrándose de nuevo en los valores que más se aproximan a las actitudes de súbdito. 


\section{Capital social y cultura política: desmontando la posible tautología}

Antes de avanzar con nuestro análisis debemos adelantarnos a una de las críticas que se pueden plantear ante nuestra propuesta de estudio: la posibilidad de que cultura política y capital social, tal y como lo hemos definido nosotros, estuviesen conectados por definición. De ser así, nuestra propuesta de estudio carecería de validez interna, por caer en una tautología. Algunos etnógrafos han, de hecho, acusado a Putnam de caer en tal error, afirmando que su comprensión de capital social se identifica con una faceta importante de la cultura, la de las conexiones interpersonales y las redes en las que el individuo se ve envuelto, por lo que la idea de capital social ya formaría, inevitablemente, parte del concepto de cultura política (Wood, 2001).

No obstante, realizar esta crítica a nuestro estudio supondría cuestionar asuntos que ya han sido clarificados con anterioridad: por una parte, se negaría la distinción sociedad civil estado/régimen democrático, por la otra, se estaría asumiendo el carácter macrosociológico del capital social.

Este razonamiento, además de resultar imposible en el marco de nuestra investigación, imposibilitaría la única estrategia que nos permitiría ahondar en un análisis exhaustivo de las causas y efectos de determinadas actitudes políticas, y es que como Almond y Verba (1970, p. 50-51) afirman "las orientaciones políticas individuales deben ser separadas analíticamente de otras clases de orientaciones psicológicas, para que realicemos 'tests' con las hipótesis acerca de la relación existente entre las actitudes políticas y otras diferentes".

Esta es la razón por la que ellos mismos, como se ha dicho antes, se proponen analizar el modo en que algunas actitudes sociales del individuo, tales como la confianza interpersonal, su participación en actividades o sus aficiones, se correlacionan o no con sus actitudes políticas (Almond y Verba, 1970, p. 299-424).

\section{INTERPRETACIÓN DE LOS RESULTADOS}

Una vez concretadas las variables con las que trabajamos resulta conveniente explicitar cuáles son nuestras hipótesis, especificando cómo se configuran en torno a ellas los fenómenos de capital social y cultura política. Así, nuestras hipótesis son:

$\mathrm{H}_{1} \quad$ Aquellos individuos que forman parte de agrupaciones de la sociedad civil, es decir, que poseen capital social cooperativo y/o estabilizador, poseen niveles de cultura política participante iguales o mayores que quienes no participan en los mismos.

$\mathrm{H}_{2}$ Distintos tipos de capital social tienen distintos efectos en la cultura política.

$\mathrm{H}_{3} \quad$ Quienes participan en grupos de estructuras abiertas, esto es, que poseen capital social inclusivo, tendrán niveles de cultura política más altos.

$\mathrm{H}_{4} \quad$ Los individuos que poseen capital social macro, es decir, cuya motivación para confiar es general, tendrán niveles de cultura política más altos que el resto. 
Ya establecidas las hipótesis con claridad y recodificadas las variables con las que contamos, las de capital social cooperativo, estabilizador y cultura política micro, así como las de sexo y educación, las cuales incluiremos en nuestro modelo como variables de control, procedemos al análisis estadístico que nos ayudará a comprender la relación existente entre las mismas.

Puesto que nuestras variables independientes son nominales y la dependiente es una variable de intervalo el enfoque que se presta a medir la relación estadística entre ambas es el análisis de regresión lineal. Este análisis nos permitirá aproximarnos a dos de los objetivos principales del uso de la estadística: determinar la existencia o no de una relación entre variables y analizar la fuerza de la misma. Desafortunadamente, la regresión no nos permitirá indagar acerca de la dirección de las relaciones dadas.

Comenzaremos estudiando las relaciones capital social cooperativo - estabilizador con la cultura política. Para ello hemos creado seis modelos, tal y como se observa en el conjunto de tablas del cuadro 5. El primer modelo dibuja la relación entre el capital social cooperativo y la cultura política micro. El segundo se centra en el papel del capital social estabilizador como variable independiente. El tercero, por su parte, considera bajo la etiqueta de "mixto" a los individuos que poseen ambos tipos de capital social, tanto el cooperativo como el estabilizador. Los siguientes modelos combinan los tres supuestos (modelo 4), y añaden las otras variables que hemos mencionado, el sexo del individuo (modelo 5) y la educación (modelo 6). ${ }^{3}$

Así, nuestro primer interés es el de determinar si existe una relación o no entre las variables elegidas. Nuestra hipótesis nula $\left(\mathrm{H}_{0}\right)$ será la de independencia, siendo la hipótesis alternativa $\left(\mathrm{H}_{1}\right)$ la de dependencia. De este modo, y tal como se aprecia en el conjunto de tablas 4, en la que los valores para $\mathrm{F}$ se alejan considerablemente del 0 y la significación es menor de 0,01 , podemos afirmar que para los seis modelos la hipótesis nula se rechaza, aceptándose la hipótesis alternativa. Afirmamos, pues, que al menos una de las variables independientes es significativa en cada modelo.

Una vez aceptada $\mathrm{H}_{1}$ podemos adentrarnos en el análisis de la fuerza de la relación que se da en cada modelo. La pregunta que se nos plantea es la siguiente: ¿cómo de grandes son los efectos principales de las variables independientes en la variable dependiente? Hallar una respuesta fiable a esto nos permitiría realizar predicciones de la variable dependiente.

Atendiendo a la razón $\mathrm{R}^{2}$ observamos que el primer modelo, que incluye como variable independiente únicamente lo que hemos denominado capital social cooperativo explica un $9,2 \%$ de la varianza en la cultura política del individuo.

El segundo modelo, que considera como variable independiente al capital social estabilizador, explica la varianza de la variable dependiente en un 3,4\%. El tercer modelo, que se refiere a la posesión de ambos tipos de capital social, explica la variable de cultura política micro en un $5,7 \%$. Al combinar estas tres aproximaciones en el cuarto modelo la capacidad explicativa aumenta hasta un 9,9\%. Así, de entre estas propuestas tomaremos el modelo 4 como el de mayor ajuste, si bien hay que tener en cuenta que los coeficientes para el capital social mixto no son estadísticamente significativos.

\footnotetext{
${ }^{3}$ Se han codificado con el valor 0 , la constante, la no posesión del capital social correspondiente. El mismo valor se ha dado para los hombres, así como para los individuos con un nivel educativo por debajo del nivel de bachillerato o FP de grado superior.
} 


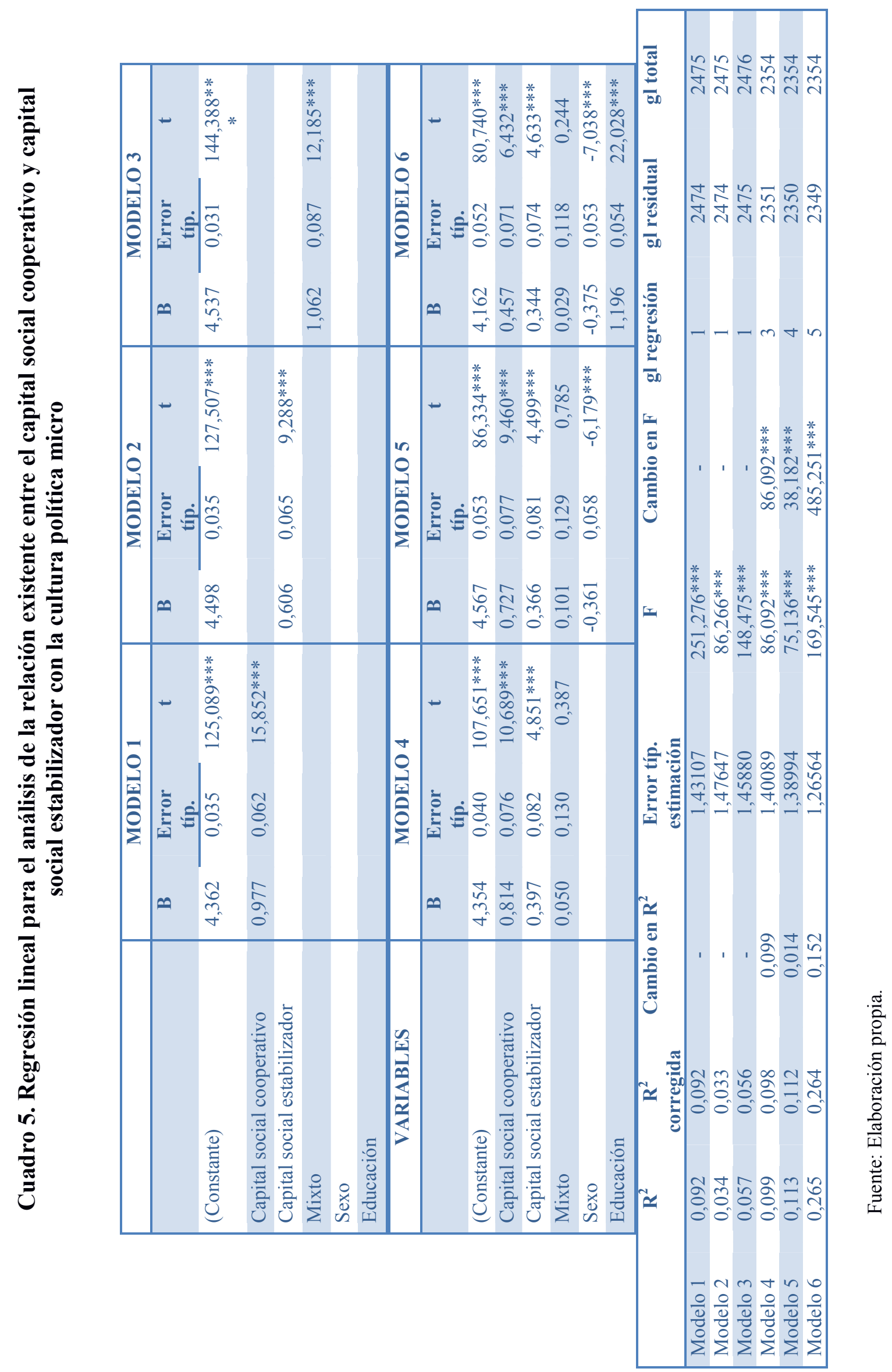


Si continuamos explorando la relación de estas variables con la variable dependiente, sometiéndolas al control de otras como, por ejemplo, el sexo del individuo, el poder explicativo del modelo se sitúa en el 11,3\%. El sexto modelo, que es el más esclarecedor, pues aumenta el poder explicativo de la relación en más de diecisiete puntos porcentuales, explica la varianza en la variable dependiente en un 26,5\%. Esto sucede porque incluye la educación. Es importante destacar también que el valor de $\mathrm{R}^{2}$ corregido no se aleja excesivamente de los anteriores porcentajes, lo cual nos asegura la fiabilidad de los resultados.

¿Qué otra información relevante aportan estos datos? Un aspecto interesante que se observa a través de este análisis de regresión es el efecto neto que tiene cada variable en distintas situaciones. Al ir añadiendo variables independientes algunos coeficientes se reducen, lo cual demuestra que el efecto de unos está recogido en cierta medida por otros. Dicho de otro modo: existe una relación entre las variables independientes. Tal hecho puede observarse con mayor precisión a través del análisis de las tablas de contingencia que las relacionan.

Por ejemplo, el coeficiente para capital social cooperativo se reduce considerablemente, de 5,294 a 4,619, al controlar la educación, lo cual nos hace pensar que ambas variables están relacionadas, lo cual queda demostrado al observar los valores para los residuos corregidos en la siguiente tabla (cuadro 6), que demuestran que la frecuencia esperada en el caso de ser ambas variables independientes, difiere enormemente de la frecuencia observada.

Cuadro 6. Relación capital social cooperativo y nivel educativo

\begin{tabular}{|c|c|c|c|c|c|}
\hline & \multicolumn{2}{|c|}{ EDUCACIÓN } & \multirow[t]{2}{*}{ Total } \\
\hline & & & $\mathbf{0}$ & 1 & \\
\hline \multirow{6}{*}{$\begin{array}{c}\text { CAPITAL } \\
\text { SOCIAL } \\
\text { COOP. }\end{array}$} & \multirow[t]{3}{*}{ No } & Recuento & 1022 & 549 & 1571 \\
\hline & & $\%$ & $76,6 \%$ & $53,8 \%$ & $66,7 \%$ \\
\hline & & Residuos corregidos & 11,6 & $-11,6$ & \\
\hline & \multirow[t]{3}{*}{ Sí } & Recuento & 313 & 471 & 784 \\
\hline & & $\%$ & $23,4 \%$ & $46,2 \%$ & $33,3 \%$ \\
\hline & & Residuos corregidos & $-11,6$ & 11,6 & \\
\hline \multirow{2}{*}{\multicolumn{2}{|c|}{ Total }} & Recuento & 1335 & 1020 & 2355 \\
\hline & & $\%$ & $100,0 \%$ & $100,0 \%$ & $100,0 \%$ \\
\hline
\end{tabular}

Fuente: Elaboración propia.

Otros cambios, aunque menos drásticos, se producen cuando se controla el sexo de los individuos, lo cual se puede explicar por la inclusión, por ejemplo, de organizaciones como sindicatos, tradicionalmente masculinizadas, a la hora de identificar el capital social estabilizador micro.

De manera análoga podemos estudiar la información que nos aporta la regresión diseñada para el capital social según su naturaleza macro y micro (cuadro 7). Creemos oportuno ahondar en esta cuestión para comprobar si, tal y como hemos asumido a lo largo de este trabajo, dicha diferencia es relevante a efectos explicativos. 


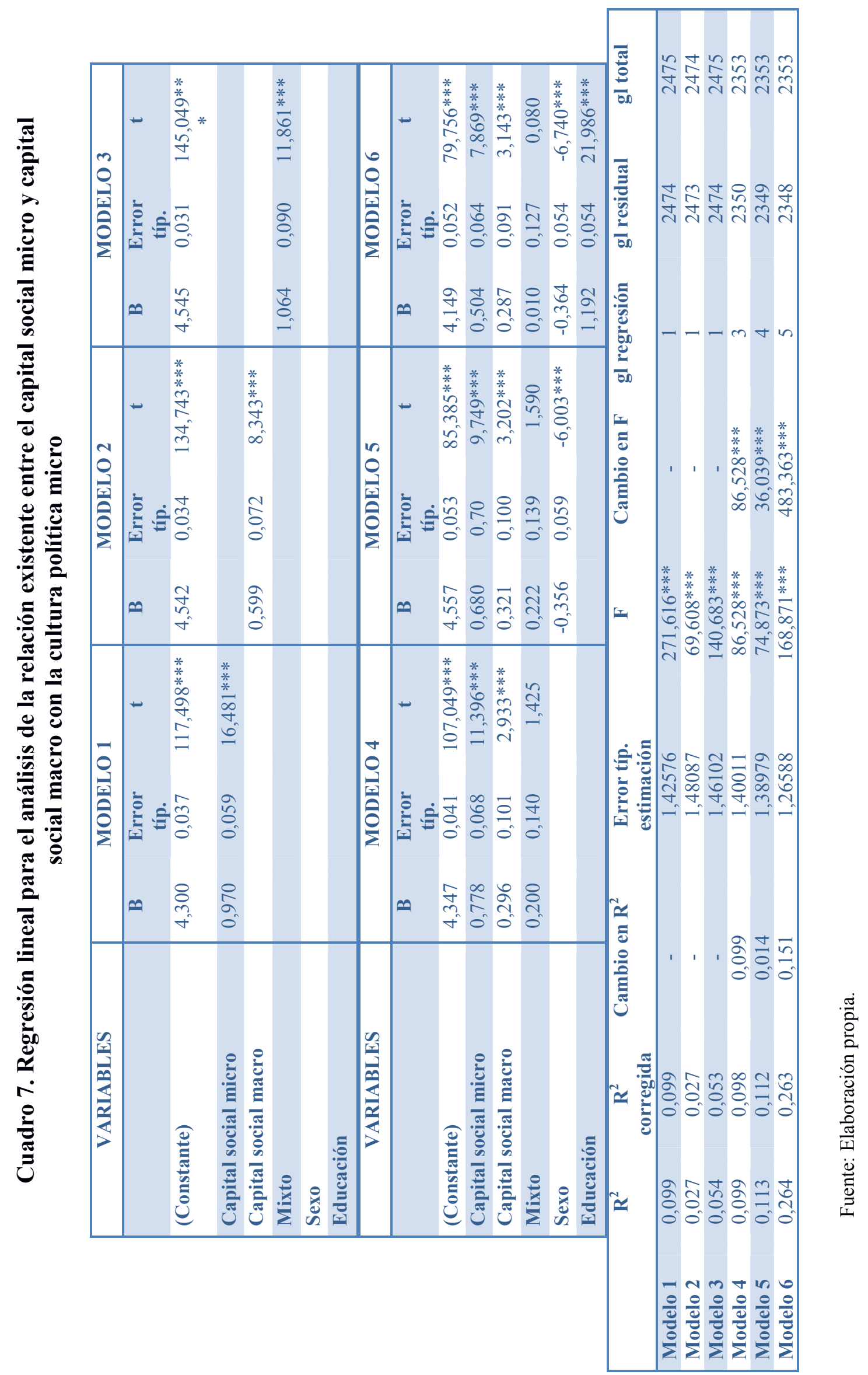


El primer paso, al igual que en el análisis anterior, es comprobar si los modelos propuestos son reveladores. Atendiendo, pues, a la razón F, podemos afirmar que en todos los modelos al menos una de las variables independientes es significativa. Es decir, existe una relación entre las variables independientes elegidas y la variable dependiente.

Al observar la razón $\mathrm{R}^{2}$, por otra parte, afirmamos que el primer modelo, el cual incluye únicamente el capital social micro como variable independiente, explica la varianza en la variable dependiente en un 9,9\%. El segundo modelo, basado en el capital social macro, la explica en un $2,7 \%$, y el tercero, teniendo como variable independiente el capital social mixto, esto es, la posesión de ambos capitales sociales, explica la varianza de la cultura política en un $5,4 \%$. Si aunamos los tres modelos en un cuarto el poder explicativo del mismo se sitúa en el $9,9 \%$. Este porcentaje aumenta según se incluyen otras variables, alcanzando el 11,3\% al tener en cuenta el sexo de los individuos y el $26,4 \%$ cuando se toma en consideración el nivel educativo. De entre estos seis modelos, pues, escogeríamos el último como el más ajustado, por ser el que explica en mayor medida los cambios en la variable dependiente.

De entre las dinámicas internas, es decir, las relaciones entre las variables independientes, destacamos la relación existente entre los propios capitales sociales macro y micro y los efectos de la educación. La inclusión del sexo del individuo como variable no produce cambios bruscos en los coeficientes. De nuevo, esta relación se hace evidente a través del análisis de las tablas de contingencia que relacionan dichas variables.

Estos dos análisis, pues, nos permiten extraer una conclusión acerca de la segunda hipótesis que planteamos, la de que distintos tipos de capital social influyen de distinto modo en los niveles de cultura política.

Los modelos presentados, tanto en la dimensión cooperativo-estabilizador como micro-macro, difieren en su poder explicativo. En el primer caso, es el modelo basado en el capital social cooperativo el que explica en mayor medida la variable dependiente, concretamente en un $9,2 \%$, frente al 3,4\% del estabilizador. En el segundo, se trata del capital social micro, con un $9,9 \%$, frente al 2,7\% del capital social macro. Estas diferencias podrían sostener la idea de que la relación de cada tipo de capital social para con la cultura política es distinta, si bien no podemos extraer en qué sentido lo es.

Lo expuesto hasta aquí es toda la información exacta que el análisis de regresión puede aportarnos. Como hemos dicho antes la dirección de la relación entre variables es una cuestión que escapa al alcance de este método. No podemos afirmar con seguridad que todos los individuos de la población poseerán los niveles de cultura política que indica la media de nuestra regresión, pues cabe la posibilidad de que, en realidad, los valores para cada individuo se sitúen en uno de los extremos del intervalo de confianza que nos proporciona el análisis estadístico. Dicho de otro modo, el no conocer con certeza la dispersión de valores a la hora de calcular la media nos impide predecir con total seguridad los valores que se encontrarán en la población.

Puesto que esta es la gran limitación de nuestro análisis, no cabe otra posibilidad que optar por la interpretación especulativa de los coeficientes B, los cuales nos indican el nivel medio de cultura política para los individuos según su tipo de capital social, para acercarnos a nuestras otras dos hipótesis. Así, extraeremos una serie de conclusiones que han de entenderse en todo momento como interpretaciones pendientes de contrastación, conformando un cuerpo 
de ideas que abren la posibilidad a nuevas investigaciones. ${ }^{4}$

De este modo la primera idea que podemos extraer es que la posesión de capital social, independientemente del tipo, supone un aumento de los niveles de cultura política del individuo, en el sentido de la cultura política participante. Atendiendo a las medias aquellos individuos que no cuentan con capital social cooperativo, estabilizador, macro o micro disponen de niveles de cultura política inferiores que quienes sí disponen de los mismos. Este hecho nos pondría en condición, pues, de afirmar la veracidad de la primera hipótesis $\left(\mathrm{H}_{1}\right)$.

Por otra parte, al comparar los coeficientes para cada una de las dimensiones, cooperativoestabilizador y micro-macro, descubrimos que la media de cultura política de aquellos que poseen capital social cooperativo es mayor que la de quienes poseen capital social estabilizador. Del mismo modo, la media para los individuos con capital social micro es mayor que los que tienen capital social macro. Todo ello independientemente de las variables con las que controlemos, sean estas el sexo del individuo, su nivel educativo o la posesión del capital social opuesto.

Así, nuestra tercera hipótesis quedaría confirmada, mientras la cuarta sería refutada.

La pregunta que surge ahora es, ¿por qué sucede esto?

Como hemos ido argumentando a lo largo de nuestro trabajo, atribuimos la existencia de niveles de cultura política más altos en individuos con capital social cooperativo a los efectos que tiene la confianza inclusiva. Es decir, entendemos que la confianza que permite la inclusión del otro, que no crea identidades excluyentes, se orienta en mayor medida a la cultura política participante por reconocer en el otro a un actor igual, por visualizar un nosotros, una colectividad, siendo ésta una noción clave en el juego democrático.

De un modo similar nuestra hipótesis inicial contaba con que el capital social macro, orientado por motivaciones generales y, por tanto, de cierto carácter colectivo, se relacionase en mayor medida que el capital social micro con la cultura política participante. Sin embargo, la razón por la que lo contrario sucede podría encontrarse en el haber utilizado como máxima expresión de este capital social el ejemplo de los sindicatos, los cuales han estado siempre envueltos en la acción política de un modo especial. Así, puesto que los sindicatos llevan a cabo numerosas acciones políticas, es de esperar que quienes forman parte de ellos posean actitudes políticas del tipo participante.

\section{CONCLUSIONES}

A través de este trabajo hemos podido aproximarnos a la cuestión de la relación entre la sociedad civil y la democracia. Habiendo hecho un recorrido por los orígenes del debate planteamos una teoría clara, basada en dos ideas, la de que (I) la existencia de la sociedad civil promueve y mantiene la democracia, por estar relacionada con la aparición de actitudes propia de la cultura política participante y que (II) ciertos sectores de la misma promueven actitudes democráticas en mayor medida que otros, en función del fin de la agrupación y del modo en que se dan las relaciones en dicha red.

\footnotetext{
${ }^{4}$ Reconocemos no obstante la posibilidad de, en futuros trabajos, aproximarnos a la comparación estadística de los coeficientes mediante la contrastación de hipótesis de restricciones lineales.
} 
De esta teoría extrajimos cuatro hipótesis:

$\mathrm{H}_{1} \quad$ Aquellos individuos que forman parte de agrupaciones de la sociedad civil, es decir, que poseen capital social cooperativo y/o estabilizador, poseen niveles de cultura política participante iguales o mayores que quienes no participan en los mismos.

$\mathrm{H}_{2} \quad$ Distintos tipos de capital social tienen distintos efectos en la cultura política.

$\mathrm{H}_{3} \quad$ Quienes participan en grupos de estructuras abiertas, esto es, que poseen capital social inclusivo, tendrán niveles de cultura política más altos.

$\mathrm{H}_{4} \quad$ Los individuos que poseen capital social macro, es decir, cuya motivación para confiar es general, tendrán niveles de cultura política más altos que el resto.

A través de un estudio estadístico, partiendo de información recogida en un barómetro del Centro de Investigaciones Sociales y reconfigurando las variables iniciales en función de nuestras necesidades hemos podido contrastar dichas hipótesis para afirmar la veracidad de las tres primeras, refutando la última.

Así, y a pesar de los límites del modelo estadístico empleado, podemos decir que nuestra teoría ha sido aprobada.

Además, hemos cumplido con nuestro propósito de ahondar en la reconstrucción del concepto de capital social, elaborando una tipología que ha permitido maximizar la utilidad teórica de este concepto.

No obstante, es necesario llamar la atención sobre los aspectos más cuestionables de nuestro trabajo, apuntando a las vías que mejorarían el mismo.

En primer lugar, es fundamental mencionar la falta de información concreta sobre los distintos tipos de capital social del individuo. La encuesta cerrada del CIS únicamente nos permitió conocer el tipo de asociación al que pertenecía cada individuo, sin entrar en detalles acerca de la motivación del sujeto a participar, el objetivo principal de la agrupación o la estructura de la misma.

Estas dificultades podrían salvarse a través de la elaboración de encuestas más específicas. Podemos mencionar, por ejemplo, el estudio de la Encuesta Mundial de Valores para el año 1990, la cual, tras preguntar al individuo sobre su participación en determinadas asociaciones, indagaba acerca de las razones que le llevaba a tomar parte en ella (World Values Survey, 2013). Añadiremos, no obstante, que elegimos no recurrir a esta encuesta por no contar la misma con información alguna que pudiera ayudarnos a medir la cultura política.

Por otra parte, un aspecto que queremos destacar es la necesidad de relativizar los efectos del capital social en la cultura política, pues no debemos pasar por alto la influencia de otros factores. No nos referimos aquí ya a elementos que han sido controlados en nuestros modelos, como el nivel educativo, el sexo o la edad del individuo, sino a elementos de contexto que enmarcan de una manera más general, y posiblemente más influyente, el modo en que interactúan los fenómenos de capital social y cultura política. Hablamos, por ejemplo, de las instituciones. 
Muchos estudios sobre capital social recalcan la importancia del contexto institucional en el análisis del mismo (van Deth et al., 1999; Heying, 2001; Newton, 2001). En nuestro caso, por ejemplo, resulta imprescindible contextualizar las dinámicas de interacción descritas en el marco del sistema político español. Esto se debe a que las instituciones condicionan, en mayor o menor medida, el modo en que los individuos se integran e interactúan en la esfera política y social. Es por esto que, muy posiblemente, de elaborar un estudio idéntico con datos pertenecientes a individuos de otro país, los resultados obtenidos diferirían de los descritos aquí.

A pesar de estos límites, sin embargo, no debemos subestimar las aportaciones de este trabajo. Con él, además de acercarnos a uno de los grandes debates de la disciplina, planteando una serie de conclusiones en el marco de nuestra teoría inicial, proponemos una nueva vía de acercamiento al análisis empírico de la sociedad civil: la de su operacionalización a través del capital social.

Creemos que esta última es, de hecho, la aportación de mayor calado de este trabajo, pues indaga acerca de una fórmula de operacionalización por la que pocos autores han optado, mostrando con claridad su potencial explicativo.

\section{BIBLIOGRAFÍA}

ALMOND, Gabriel y VerBA, Sidney. La cultura cívica: estudio sobre la participación política democrática en cinco naciones. Madrid: Euroamerica, 1970.

Bourdieu, Pierre. The Forms of Capital. En: Richardson, J.G. (Ed.). Handbook of Theory and Research for the Sociolgy of Education. New York: Greenwood Press, 1986, p. 241-258.

BouRdieu, Pierre. La distinción: criterios y bases sociales del gusto. Madrid: Taurus, 1998.

BOUZA ÁlVAREZ, Fermín. Incompetencia, corrupción y desafección ciudadana en el caso español. $2^{a}$ Tribuna de Debate Fundación Araguaney, Santiago de Compostela, 11 de marzo de 2013.

Bowler, Shaun; Donovan, Todd y Hanneman, Robert, Art for Democracy's Sake? Group Membership and Political Engagement in Europe. The Journal of Politics, 2003, vol. 65, $\mathrm{n}^{\circ} .4$ (Nov.), p. 1111-1129.

BREHM, John y RAHN, Wendy. Individual-Level Evidence for the Causes and Consequences of Social Capital. American Journal of Political Science, 1997, vol. 41, no 3, p. 999-1023.

BurT, R. S. Structural Holes: the Social Structure of Competition. Cambridge: Harvard University Press, 1992.

CAlvo Muñoz, Montserrat y RoJas Llamas, Carolina. Networking: uso práctico de las redes sociales. Madrid: ESIC, 2009.

CIS - Centro De InVestigaciones SociológiCAS. Estudios $n^{\circ} 2960$ y $n^{\circ} 3013$, accesibles a 
través de [http://www.cis.es/cis/opencm/ES/11_barometros/index.jsp], consultado por última vez el 14 de enero de 2014.

Cohen, Jean L. y ARATo, Andrew. Sociedad civil y teoría política. México: Fondo de Cultura Económica, 2000.

Coleman, James S. Social Capital in the Creation of Human Capital. American Journal of Sociology, 1988, vol. 94, Supplement: Organizations and Institutions: Sociological and Economic Approaches to the Analysis of Social Structure, (p. S95-S120)

Coleman, James S. Foundations of Social Theory. Massachusetts: First Harvard University Press, 1994

FARR, James. Social Capital: a Conceptual History. Political Theory, 2004, vol. $32 \mathrm{n}^{\mathrm{o}} 1$, February. p. 6-33.

Gambetta, Diego. Can We Trust Trust?. En: GambettA, D. (Ed). Trust: Making and Breaking Cooperative Relations. Cornwall: Basil Blackwell, 1990.

Granovetter, Mark S. The Strength of Weak Ties. American Journal of Sociology, 1973, vol. 78, Issue 6 (May), p. 1360-1380.

Hegel, Guillermo Federico. Filosofía del Derecho. Buenos Aires: Claridad, 1968.

HEYING, Charles H. Civic elites and corporate delocalization: an alternative explanation for declining civic engagement. En: EDWARDS, Bob et al. Beyond Tocqueville: Civil Society and the Social Capital Debate in Comparative Perspective. Hanover: University Press of New England, 2001, p.101-110.

JACOBS, Jane. Muerte y vida de las grandes ciudades. Madrid: Península, 1967.

Kadushin, Charles. Understanding Social Networks: Theories, Concepts and Findings. New York: Oxford University Press, 2012.

Lewis, J. David y Weigert, Andrew. Trust as a social reality. Social Forces, 1985, vol. 6, no 4, p. 967-985.

Luhmann, Niklas. Trust and Power. Avon: Pitman Press, 1979.

Misztal, Barbara A. Trust in Modern Societies: the search for the bases of social order. Cornwall: Polity Press, 1996.

MONTERO, José Ramón y TORCAL, Mariano. "Facets of social capital in new democracies: the formation and consequences of social capital in Spain", en VAN DETH, Jan W. et al. (Eds). Social Capital and European Democracy. London: Routledge-ECPR, 1999, p. 154-176.

Montero, José Ramón y TorCal, Mariano. No es el descontento, es la desafección. Diario El País, 14 de junio de 2013, accesible a través de [http://elpais.com/elpais/2013/06/11/opinion/1370978768_828729.html], consultado por última vez el 25 de junio de 2013. 
Moreno Feliu, Paz. Encrucijadas antropológicas. Madrid: Universitaria Ramón ArecesUNED, 2010.

Newton, Kenneth. Social capital and democracy. En: EDwARDS, Bob et al. Beyond Tocqueville: Civil Society and the Social Capital Debate in Comparative Perspective. Hanover: University Press of New England, 2001, p. 225-233

Newton, Kenneth y Norris, Pippa. Confidence in Public Institutions: Faith, Culture or Performance? En: Pharr, Susan y Putnam, Robert D. (Eds.). Disaffected Democracies: What's Troubling the Trilateral Countries? Princeton, NJ: Princeton University Press, 2000.

Ostrom, Elinor y Ahn, T. K. Una perspectiva del capital social desde las ciencias sociales: capital social y acción colectiva. Revista Mexicana de Sociología, 2003, Año 65, no 1, EneroMarzo.

Ostrom, Elinor y WALKeR, James (Eds.). Trust and Reciprocity: Interdisciplinary Lessons from Experimental Research. New York: Russell Sage Foundation, 2005.

PaXton, Pamela. Is Social Capital Declining in the United States? A Multiple Indicator Assessment. American Journal of Sociology, 1999, vol. 105, nº 1, p. 88-127.

PaXton, Pamela. Social Capital and Democracy: an Interdependent Relationship. American Sociological Review, 2002, vol. 67, April, p.254-277.

PEÑA, Javier. La sociedad civil. En: ARTETA, Aurelio et al. (Ed). Teoría política: poder, moral, democracia, Madrid: Alianza, 2003, pp. 196-210.

PorTes, Alejandro. Social Capital: its Origins and Applications in Modern Sociology. Annual Review of Sociology, 1998, vol. 24, p. 1-24.

PUTNAM, Robert D. Making democracy work: civic traditions in modern Italy. New Jersey: Princeton University Press, 1993.

Putnam, Robert D. Turning In, Turning Out: the Strange Disappearance of Social Capital in America. PS: Political Science and Politics, 1995, vol. 28, nº 4 (Dec.), p. 664-683.

PutNAM, Robert D. Solo en la bolera: colapso y resurgimiento de la sociedad norteamericana. Barcelona: Galaxia Gutemberg-Círculo de Lectores, 2002.

Quintellier, Ellen. Who is Politically Active: the Athlete, the Scout Member or the Environmental Activist? Young People, Voluntary Engagement and Political Participation. Acta Sociologica, 2008. vol. 51, nº. 4, p. 355-370.

Rebola PARdo, Adrián et al. Social Network Communities Characterization as Density Local Maxima. Granada: Universidad de Granada. [pendiente de publicación], 2013.

SNIJDERS, Chris. Review: Trust in Modern Societies: the Search for the Bases of Social Order. American Journal of Sociology, 1997. vol. 102, nº 6, p. 1724-1729. 
TENZER, Nicolas. La sociedad despolitizada. Barcelona: Paidós, 1992.

TocQueville, Alexis de. La Democracia en América. Barcelona: Tecnos, [1835] (ed. 2011).

USLANER, Eric M. Morality plays: social capital and moral behaviour in Anglo-American democracias. En: Jan W. van Deth, Marco Maraffi, Ken Newton y Paul F. Whiteley (Eds). Social Capital and European Democracy. London: Routledge-ECPR, 1999, p. 213-239.

van Deth, Jan W.; Maraffi, Marco; Newton, Ken; Whiteley, Paul F. Social Capital and European Democracy. London: Routledge-ECPR, 1999.

VÁzQUez García, Rafael. Compromiso cívico y democracia: los efectos democráticos del asociacionismo sociopolítico en España. Sevilla: Centro de Estudios Andaluces, 2010.

Warren, Mark E. Democracy and Association. New Jersey: Princeton University Press, 2001.

Whittington, Keith. Revisiting Tocqueville's America: Society, Politics and Association in the Nineteenth Century. En: EdWARDS, Bob et al. Beyond Tocqueville: Civil Society and the Social Capital Debate in Comparative Perspective. Hanover: University Press of New England, 2001, p. 21-31

Williams, Bernard. Formal structures and social reality. En: GAMBETTA, D. (Ed). Trust: Making and Breaking Cooperative Relations. Cornwall: Basil Blackwell, 1990, p. 3-13.

WooD, Richard L. Political Culture Reconsidered: Insights on Social Capital from an Ethnography of Faith-Based Community Organizing. En: EDWARDS, Bob et al. Beyond Tocqueville: Civil Society and the Social Capital Debate in Comparative Perspective. Hanover: University Press of New England, 2001, p. 254-265.

WORLD VAlues SuRVEY. 1990 Questionnaire, accesible a través de [http://www.worldvaluessurvey.org/wvs/articles/folder_published/survey_1990/files/ root_q1990.pdf], consultado por última vez el 24 de mayo de 2013.

(C) Copyright Rocío Saavedra Fernández, 2015.

(C) Copyright GeoGraphos, 2015.
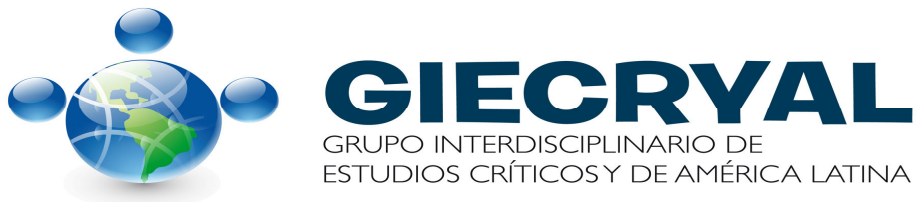